\title{
The Pursuit of Neutrality in the Metaphysics of Emergence
}

\author{
Umut Baysan \\ University of Oxford \\ umut.baysan@philosophy.ox.ac.uk
}

\section{Introduction}

The world seemingly contains emergence. On the face of it, the natural world is populated by macro-level entities, such as rocks, houses, humans, and milk bottles. These macro-level entities are made up of micro-level physical entities that co-exist with them: humans are made up of cells and milk bottles are made up of milk bottle parts. Various features, i.e., properties and relations (henceforth, simply properties), that these macro-level entities bear are dependent on various configurations of the micro-level entities that make them up and the properties of these micro-level entities: the size and the shape of a house are dependent on the sizes and the shapes of its parts, and how they are put together. This much is not that controversial - at least for those who are realists about things like houses and milk bottles, and, to some extent, the properties we attribute to such things. But more controversially-and this is what marks emergence as a metaphysically interesting idea-many macro-level entities and their properties are ontologically and causally autonomous in relation to the micro-level entities and properties they depend on. That is, humans are not reducible to cells (ontological autonomy), and the things we can say about the causal powers of a rock may be very different from the things we can say about the causal powers of the solid crystals that make up a rock (causal autonomy). Or so argues Jessica Wilson, very thoroughly and (in my opinion) convincingly, in Metaphysical Emergence (2021). To do so, she adopts a "metaphysically highly neutral" (p. 32) approach to questions about powers, causation, properties, and laws. That is, while explaining what emergence is and arguing that there is indeed emergence in the natural world, she doesn't restrict her analyses and arguments to any specific metaphysical accounts of these concepts, and she doesn't commit to any controversial theses about them. In what follows, I assess Wilson's project against this methodological ideal of metaphysical neutrality.

In $§ 1$, I introduce Wilson's central distinction between weak emergence and strong emergence, explain how these concepts relate to physicalism, and make her metaphysically neutral methodology explicit. In $\S 2$, after explaining how Wilson uses this distinction to solve the problem of higher-level causation, I argue that her commitment to metaphysical neutrality regarding causal concepts comes with a potential cost: often different theories regarding these notions make different predictions in identifying the causes or effects of an event, and this leads to a worry that, in Wilson's framework, the truth (or falsehood) of physicalism ultimately turns on what account(s) of these notions we ought to employ. In $\S 3$, I show that there are points where I think Wilson departs from her ideal of metaphysical neutrality. This seems to be the case when she dismisses (putative) non-causal aspects of properties (e.g., 
quiddities), which some accounts that are relevant to the debate on emergence evidently posit. In $\S 4$, I relate these worries to epiphenomenalism, which is a view that finds no room in Wilson's project.

\section{Wilson on Metaphysical Emergence}

For Wilson, ontological autonomy and causal autonomy (of dependent entities) are the marks of emergence. The idea behind ontological autonomy is straightforward. Focusing on properties, we can understand ontological autonomy as irreducibility of the properties in question. Insofar as macro-level properties are not identical with micro-level properties (or arrangements thereof), emergent properties satisfy the condition on ontological autonomy. There are well-known arguments for making a case for ontological autonomy (e.g., including the argument from the "multiple realizability" of higher-level properties), and I will not revisit them here.

Causal autonomy is a little less straightforward and it requires some detailed spelling-out. Wilson takes causal autonomy to be something along the lines of "distinctive efficacy" (p. 1). A property $\mathrm{P}$ is causally autonomous from another property $\mathrm{Q}$ insofar as $\mathrm{P}$ is distinctively causally efficacious vis-à-vis $\mathrm{Q}$ - it has a different/distinctive causal profile, so to speak. Wilson goes on to argue that many higher-level properties that are characteristic of the special sciences (such as biology, geology, meteorology, psychology) are causally autonomous in this sense in relation to lower-level physical properties. For Wilson, the relevant kind of distinctive efficacy comes with a twofold distinction, and this distinction is crucial for Wilson's project.

On the one hand, higher-level properties may have causal powers that do not go beyond-and form a proper subset of - the causal powers of the micro-level properties they depend on. For example, one might think that the causal powers that we attribute to a pain experience, such as priming the subject to exhibit pain behaviour, may be a proper subset of the causal powers that we attribute to the neurophysiological state that underlies that pain experience. Let's suppose that the neurophysiological state in question is C-fibre activation. Then, every causal power of a pain experience may also be a causal power of $\mathrm{C}$-fibre activation, but $\mathrm{C}$-fibre activation may have some causal powers that the pain experience does not have, for example to produce a specific reading on a neuron detector. Generalising from this example, and on the assumption that, ultimately, the relevant base properties are micro-level physical properties - i.e., they are treated by physics and are "not fundamentally mental" (p. 23) - the causal powers of the relevant special-science properties form a proper subset of the causal powers of physical properties.

For Wilson, this is characteristic of weak emergence. That is, emergent properties that have only a proper subset of the causal powers of the physical properties they depend on are weakly emergent properties. Given that the relevant micro-level properties are physical properties, if properties that are characteristic of a given special science are weakly emergent in Wilson's sense, then physicalism about that special science must be true. In fact, this would be physicalism of a non-reductive kind, as these properties will be non-identical with the physical properties they depend on (for reasons to do with, inter alia, multiple realizability). This gives us the so-called "proper subset condition" for weak emergence: 
What it is for token feature $\mathrm{S}$ to be Weakly metaphysically emergent from token feature $\mathrm{P}$ on a given occasion is for it to be the case, on that occasion, (i) that $\mathrm{S}$ cotemporally materially depends on $\mathrm{P}$, and (ii) that $\mathrm{S}$ has a non-empty proper subset of the token powers had by $\mathrm{P}$. (p. 72)

On the other hand, a special-science property may have causal powers that go beyond the causal powers of the physical property/properties that it depends on. Going back to our example about pain, perhaps a pain experience has some causal powers that C-fibre activation doesn't have. For Wilson, this is characteristic of what she calls strong emergence. Thus, if pain has novel causal powers compared to its base-level properties, then pain is strongly emergent. This "new power condition" for strong emergence is thus understood as follows:

What it is for token feature $\mathrm{S}$ to be Strongly metaphysically emergent from token feature $\mathrm{P}$ on a given occasion is for it to be the case, on that occasion, (i) that $\mathrm{S}$ cotemporally materially depends on $\mathrm{P}$, and (ii) that $\mathrm{S}$ has at least one token power not identical with any token power of P. (p. 53)

For Wilson, strong emergence of a property is incompatible with physicalism (about the property in question) (p. 41, p. 54, and elsewhere). This is very plausible. A key tenet of physicalism is the principle that is known as Physical Causal Closure: any physical event that has a cause has a sufficient physical cause. But if the causal powers of an emergent property go beyond those of the physical property/properties it depends on, then presumably there will be events - including physical events - that have causes without thereby having sufficient physical causes, meaning that Physical Causal Closure will be false (p. 44).

In case it is not clear from what has been said already, let me note that understanding weak emergence, strong emergence, and how these two are related to physicalism in terms of causal powers differs from alternative accounts whereby these concepts are understood in terms of either supervenience or explanation (or a combination of these two). ${ }^{1}$ Consequently, Wilson departs from understanding physicalism merely in terms of a supervenience thesis. ${ }^{2}$

The distinction between weak emergence and strong emergence is crucial to Wilson's project (both in Metaphysical Emergence and in her previous work, e.g., her 2015). After articulating (weak and strong) emergence in this fashion, Wilson goes on to explore a series of interesting questions. These include: Do complex systems and their behaviours (e.g., flocking behaviours of birds) give us cases of weak emergence or strong emergence? (Chapter 5) Are ordinary objects (e.g., natural objects like rocks and artefacts like tables) weakly emergent or strongly emergent? (Chapter 6) Is consciousness weakly emergent or strongly emergent? (Chapter 7) On the assumption that we have genuine free will, is the exercise of such free will a case of weak emergence or strong emergence? (Chapter 8) With the exception of the case of free will, Wilson opts for weak emergence. When it comes to free will, Wilson argues that the kind of free will that we intuitively have - the kind that is incompatible with determinism - supports the idea that our exercise of free will may be case of strong emergence. Ultimately, most of these questions are about the truth of physicalism. This is because while the strongly emergent properties are "physically unacceptable" properties (p. 54), the weakly emergent ones are "physically acceptable" properties (p. 73). So, if Wilson is

\footnotetext{
${ }^{1}$ See, among others, Noordhof (2003) and Chalmers (2006) for such alternative accounts.

${ }^{2}$ See Wilson (2005) for problems with supervenience-based formulations of physicalism.
} 
right about free will, physicalism may be false! But, contrary to a commonly shared viewed, consciousness doesn't pose a serious problem for physicalism.

More importantly, if Wilson is right about the relationship between physicalism and weak/strong emergence, it follows that whether physicalism is true or not ultimately turns on the question of what we ought to say about the relationship between the causal powers we attribute to special-science properties on the one hand, and the causal powers we attribute to physical properties on the other hand. And this takes us to a central question in metaphysics of science: How should we make sense of the relationship between properties and causal powers?

Wilson clarifies what she means by powers:

[T]alk of powers is simply shorthand for talk of what causal contributions possession of a given feature [e.g., a property] makes (or can make, relative to the same laws of nature) to an entity's bringing about an effect, when in circumstances. That features are associated with actual or potential causal contributions ("powers") reflects the uncontroversial fact that what entities do (can do, relative to the same laws of nature) depends on how they are (what features they have). (p. 32)

Crucially, this "operative notion of 'power' ... is metaphysically highly neutral" (ibid., emphasis added):

Anyone who accepts that the effects an entity causes (or can cause, relative to the same laws of nature) are in part a function of what features the entity has - effectively, all participants to the present debate - is in position to accept "powers", in the shorthand, metaphysically neutral and nomologically motivated sense at issue here. (p. 45)

The same goes for causation:

[T] he operative notion of causation ... is also metaphysically highly neutral. ... [T] here are many specific accounts of this notion, but it may serve as an initial proof of concept ... that even a contingentist categoricalist Humean - someone who thinks that causation is a matter of regularities, such that features have their powers contingently, and that all features are ultimately categorical — can accept powers and the associated notion of causation in the neutral sense(s) here. (p. 33)

Wilson adds: "More generally, no controversial theses pertaining to the nature of powers, causation, properties, or laws are here presupposed" (p. 33).

There is much to be admired in this commitment to metaphysical neutrality regarding the notions of powers, causation, properties, and laws. That said, in what follows, I will argue that this ideal of metaphysical neutrality generates some difficulties for Wilson's project.

\section{Physicalism and Metaphysical Neutrality about Causation}

Is being "metaphysically highly neutral" about the notions of powers, causation, properties, and laws a good thing for theorising about emergence? To appreciate the importance of this question, let me first illustrate how Wilson applies her account of emergence to the problem of higher-level causation. As Wilson notes, "six premises lead to the problem of higher-level causation" (p. 40). These are:

\footnotetext{
${ }^{3}$ This is originally a passage from Wilson (2015, p. 354).
} 
1. Dependence. Special-science features cotemporally materially depend on lower-level physical features (henceforth, "base features") in such a way that, at a minimum, the occurrence of a given special-science feature on a given occasion minimally nomologically supervenes on base features on that occasion.

2. Reality. Both special-science features and their base features are real.

3. Efficacy. Special-science features are causally efficacious.

4. Distinctness. Special-science features are distinct from their base features.

5. Physical Causal Closure. Every lower-level physical effect has a sufficient purely lowerlevel physical cause.

6. Non-overdetermination. With the exception of the double-rock-throw variety [i.e., in rare cases where, e.g., two rocks independently and simultaneously hit a glass, causing the glass to break], effects are not causally overdetermined by distinct individually sufficient cotemporal causes. (p. 41, text and formatting slightly altered)

This takes us to the well-known "causal exclusion" problem that we associate with Jaegwon Kim (1998 and elsewhere). In a nutshell, the problem is this: if special-science properties are real (as per 2) but distinct (as per 4) from the base-level physical properties that they depend on (as per 1), and the latter are causally sufficient for their putative effects (as per 5), it is not clear how the former can be causally efficacious (as per 3 ) without violating the nonoverdetermination principle (as per 6).

Wilson makes a persuasive case that this problem has a satisfactory solution - at least for those who take emergence seriously. While those who take special-science properties to be strongly emergent can reject Physical Causal Closure, those who take them to be weakly emergent can reject Non-overdetermination (p. 44). I have already touched on why strong emergence (as understood in terms of the new power condition) contradicts Physical Causal Closure in $\S 1$. As for weak emergence, if we follow the proper subset condition for understanding this notion (as explained in $\S 1$ above), we have an intelligible way of rejecting Non-overdetermination. If, on a given occasion, a weakly emergent special-science property's causal powers are token-identical with those of the base-level physical property in question, we can allow this to be a case of causal overdetermination, "but maintain that the overdetermination here is of an unproblematic ... variety" (ibid.).

Let's take $\mathrm{S}$ to be our emergent property, $\mathrm{P}$ to be the base-level physical property that $\mathrm{S}$ depends on, and $\mathrm{P}^{*}$ to be a putative physical effect of $\mathrm{S}$. While the causal exclusion problem suggests that $\mathrm{S}$ can't be causally efficacious in bringing about $\mathrm{P}^{*}$-because it would be causally excluded by $\mathrm{P}$, which is already causally sufficient for $\mathrm{P} *$-Wilson's response is that $\mathrm{S}$ can be causally efficacious in relation to $\mathrm{P}^{*}$. On the one hand, if $\mathrm{S}$ is a weakly emergent property, its causal power to bring about $\mathrm{P}^{*}$ will be token-identical with $\mathrm{P}$ 's causal power to bring about $\mathrm{P}^{*}$. So, while $\mathrm{P} *$ is causally overdetermined by $\mathrm{S}$ and $\mathrm{P}$, this is not a problematic case of causal overdetermination. Call this Option 1. On the other hand, if $\mathrm{S}$ is a strongly emergent property, it may have the causal power to bring about $\mathrm{P}^{*}$ while $\mathrm{P}$ lacks this causal power, in which case causal overdetermination is avoided because Physical Causal Closure is false. Call this Option 2.

If Option 1 is the right reading of the situation at hand, then physicalism about $\mathrm{S}$ is true. This is because we have said that weak emergence is physically acceptable (insofar as the baselevel properties are physical properties). However, if Option 2 is the right reading, then physicalism must be false, as we have said that strong emergence is physically unacceptable. 
But how do we decide which of these options is the right one? That is, how do we decide if $\mathrm{S}$ or $\mathrm{P}$ (or both, or neither for that matter) is causally efficacious in relation to $\mathrm{P}^{*}$ ? My claim is that this decision is not possible without making theoretical commitments about what we take the relationship between powers, causation, properties, and laws to be. And the theoretical commitments we make inevitably play a crucial role in what answer we get.

A very easy way of making this point would be just pointing out that a theory of causation on which Non-overdetermination is true strictly rules out Option 1. Among the six premises leading to the problem of higher-level causation, the Non-overdetermination premise is the only one that makes a general claim about causation, and it can naturally be seen as a highly general theory of causation - perhaps a plausible one, perhaps not. For example, Kim, who we associate the problem of higher-level causation with, takes Non-overdetermination to be "virtually an analytical truth" about causation (2003, p. 163). So, Kim's account of causation-whatever its details may be-rules out Option 1.

A more interesting way of making the point that theoretical commitments about causal efficacy play a role in determining what to think about physicalism would be to find two toy examples of accounts of causal efficacy and show that they generate radically different results from the very same set-up (e.g., one supporting Option 1, the other Option 2). To fix ideas - and simplifying things for reasons of space - suppose we adopt a version of the "difference-making" account of causation that is defended by Christian List and Peter Menzies in the mental causation debate, and accordingly define causes as difference-makers in the following way:

The presence of $F$ makes a difference to the presence of $G$ in the actual situation just in case (i) if any relevantly similar possible situation instantiates F, it instantiates G; and (ii) if any relevantly similar possible situation instantiates not-F, it instantiates not-G. (2009, p. 482)

A result of applying this conception of causation to the problem of higher-level causation is that in cases where special-science properties are multiply realizable (i.e., effectively in all relevant cases), $\mathrm{S}$ comes out as a cause of $\mathrm{P}^{*}$ while $\mathrm{P}$ fails to come out as a cause of $\mathrm{P}^{*}$. That is, while $\mathrm{P}^{*}$ counterfactually depends on $\mathrm{S}$, it doesn't counterfactually depend on P. Due to multiple realizability of $\mathrm{S}$ by $\mathrm{P}$ and other physical properties, there are relevantly similar possible situations where $\mathrm{P}$ is not instantiated, while both $\mathrm{S}$ is instantiated and $\mathrm{P}^{*}$ occurs. For example, suppose that my pain experience is followed by my display of discomfort. Although in the actual world my $\mathrm{C}$-fibre activation realizes my pain, in a nearly possible world, a slightly different physical property may realize my pain. Plausibly, in both cases - i.e., both in the actual world in this nearby counterfactual world, my pain experience is followed by the same display of discomfort. This suggests that my display of comfort doesn't counterfactually depend on my C-fibre activation, while it counterfactually depends on my pain experience. If we follow Wilson, that should give us a case of strong emergence, falsifying physicalism about pain.

Alternatively, suppose - again just to fix ideas - we adopt an account about the relationship between properties and causal powers that I have proposed elsewhere: "A property $\mathrm{F}$ has a causal power $\mathrm{C}$ if and only if, as a matter of nomological necessity, all bearers of $\mathrm{F}$ have $\mathrm{C}$ " (Baysan 2018, p. 424). As I have argued in that work, a result of this framework is that, in relevant cases (i.e., cases where $\mathrm{P}$ nomologically necessitates $\mathrm{S}$ ), $\mathrm{S}$ and $\mathrm{P}$ can both be causally 
efficacious in relation to $\mathrm{P}^{*}$. If we follow Wilson, that should give us a case of weak emergence, verifying physicalism about $\mathrm{S}$.

It is not my aim here to assess either of these accounts of causal efficacy, thus the details of these accounts and the relevant arguments are not important for our purposes here. The observation I want to make is that being highly metaphysically neutral about the concepts of causal powers, causation, properties, and laws leads to some level of indeterminacy on what to think about physicalism.

To be fair to Wilson, she does foresee a problem along these lines when she suggests that weak emergentists should reject the "downwards exclusion" claim that follows from List and Menzies's account of causation as difference-making (pp. 81-82). But this is to say that the type of view defended by List and Menzies is not compatible with weak emergence, ${ }^{4}$ and my claim here is that this requires one to make commitments regarding causation, which means an inevitable departure from the ideal of metaphysical neutrality.

Throughout the book, Wilson is impressively thorough in responding objections that others have raised against her previous work (as well as to objections to weak and strong emergence more generally), so I would not at all be surprised if she has something informative and interesting to say in response to this worry. Regardless, I take the question of how much metaphysical neutrality is desired (and how much is too much) interesting and relevant to Wilson's project, hence I find it worth raising.

\section{Metaphysical Neutrality and Quiddities}

I now want to move on to the question of to what extent Wilson's project remains metaphysically neutral regarding the relevant notions. There is reason to think that Wilson departs from this ideal at certain points, which of course is fine (and inevitable) if I am right in my argument in $\S 2$.

Chapter 3 includes an instructive discussion of whether conforming to Wilson's schema for weak emergence is sufficient for physicalism. As part of this - and building on a debate Wilson previously had with Andrew Melnyk (see Melnyk 2006 and Wilson 2011)—Wilson discusses the objection that a special-science property $\mathrm{S}$ may conform to the proper subset condition on causal powers and may still be physically unacceptable (pp. 94-97). The objection goes as follows. S may, on a given occasion, (i) depend on $\mathrm{P}$ (which is a physical property), (ii) have only a proper subset of the causal powers of $\mathrm{P}$, but (iii) may have some non-causal "quiddity" that happens to be physically unacceptable. A quiddity is a non-causal aspect of a property: if properties have (or are) quiddities, this implies that properties are in principle separable from their causal roles. This can be seen as a dilemma: either the proper subset condition on causal powers does not guarantee physical acceptability, or Wilson must impose a ban on non-causal quiddities, in which case metaphysical neutrality is compromised.

Wilson's response to this objection is that the weak emergentist does not have to be cornered into this dilemma, as even if properties have non-causal quiddities, such quiddities bear no

\footnotetext{
${ }^{4}$ Relatedly, in previous work, Jonas Christensen and I argued for the claim that the List-Menzies view is not compatible with non-reductive physicalism (Christensen \& Baysan 2018).
} 
relevance to the present debate. The debate on metaphysical emergence is a debate on special-science properties, and

the individuation of scientific features is neutral on the presence or absence of quiddities: in scientific contexts, the occurrence of scientific features, and any truths about such features, does not depend on or otherwise track whether such features have quiddities, much less track how the noncausal quiddities of seemingly distinct features are related... As such, the Weak emergentist can reasonably maintain that whether $\mathrm{S}$ and/or $\mathrm{P}$ have quiddities ... is irrelevant to whether $\mathrm{S}$ is physically acceptable. (p. 96)

I am not entirely satisfied with this response. I think we can imagine a scenario where quiddities are relevant to the debate on metaphysical emergence. Moreover, such a scenario may be a case where $\mathrm{S}$ conforms to the proper subset condition but is nevertheless physically unacceptable.

If any issue is relevant to metaphysical emergence, the issue of phenomenal consciousness is. Wilson should agree, as she spends an entire chapter in discussing whether phenomenal consciousness is weakly or strongly emergent (ultimately arguing that it is weakly emergent). But in certain views in the metaphysics of consciousness, quiddities are relevant to phenomenal consciousness. What I have in mind is the view on which fundamental categorical properties - which are often taken to be quiddities - that ground dispositions of fundamental physical particles also have a role in grounding phenomenal consciousness of creatures like us. Inspired by Bertrand Russell's views about the nature of physics, this view is sometimes called Russellian monism. It is a form of monism because it doesn't posit distinct properties to explain physical causal powers and phenomenal consciousness.

Now, on Russellian monism, the base-level properties that ground phenomenal consciousness may or may not be physical properties in Wilson's sense of the term "physical", i.e., not fundamentally mental (p. 23). In some versions, e.g., in Russellian panpsychism, these baselevel properties are phenomenal properties (as well as being physical properties), in which case they don't pass Wilson's test for being physical. So, such views are indeed irrelevant to the considerations in question. But in other versions, the base-level properties are not phenomenal properties; they are protophenomenal properties. While protophenomenal properties are not phenomenal properties (because there is nothing it is like to have a protophenomenal property), they have a crucial role in explaining consciousness, as they intelligibly ground phenomenal consciousness. That is, from the knowledge of protophenomenal truths, the knowledge of phenomenal truths is analytically derivable. This gives us Russellian panprotopsychism (Chalmers 2015).

My argument is that such a view can be shown to conform to the proper subset condition on causal powers (and hence to weak emergence), but it is questionable that it is a physicalist view, as it is dubious that the relevant emergent properties are physically acceptable. To appreciate this point, let's take $S$ to be a phenomenally conscious property of a person, e.g., a colour experience, and $\mathrm{P}$ to be its base-level physical property, which, on the view in question, will be a micro-arrangement of fundamental physical quiddities. Let's further assume that the causal powers of S are a proper subset of the causal powers of P. Crucially, on this view, although $\mathrm{P}$ itself is a physically acceptable property (in being a fundamentally non-mental physical property), $\mathrm{S}$ is not a physically acceptable property as this is a view whereby physicalism is false. As David Chalmers-someone who takes this view to be one 
of the main contenders for solving the mind-body problem — puts it, "while the view arguably fits the letter of materialism, it shares the spirit of antimaterialism" (2003, p. 131). ${ }^{5}$

I think there are at least two ways this issue can be addressed. First, one might want to give up on the hopes of metaphysical neutrality and rule out views whereby properties have quiddities. This option is clearly open to Wilson, and she comes close to taking it. But when she says that quiddities are irrelevant to the debates in question, I think she is mistaken, as we have seen that there are relevant views that posit such quiddities. Second, contra Chalmers, we might want to reject the proposal that Russellian panprotopsychism "shares the spirit of antimaterialism". That itself would be an interesting result, and it would be a point worth making. Russellian monist views (Russellian panprotopsychism being an example) have received much attraction in recent years and examining such theories with the help of the tools that Wilson provides in Metaphysical Emergence can only lead to progress in this debate.

\section{Epiphenomenalism}

The final question I want to address naturally follows from the discussion of quiddities as non-causal aspects of properties: Is there any room for epiphenomenalism in Wilson's system? In philosophy of mind, epiphenomenalism is often taken to be the view that at least some mental properties are causally inefficacious with respect to physical effects (though they are themselves caused and sustained by physical properties/events). More generally in metaphysics of the special sciences, we can understand epiphenomenalism as the view that some special-science properties are causally inefficacious. ${ }^{6}$

Unsurprisingly, Wilson's characterisation of emergence rules out any emergent property as epiphenomenal (i.e., causally inefficacious), as she takes emergence to be a causal concept from the very outset: emergent properties are causally autonomous because they are distinctively causally efficacious. That said, although it is understandable that Wilson's two schemas for emergence don't cover epiphenomenalism, there is a worry that there is no logical space for epiphenomenalism in Wilson's metaphysics. This worry is salient when Wilson equates physical unacceptability of a special-science property with its causal novelty (as per the new power condition). But this has the implication that epiphenomenalism about special-science properties is not a physically unacceptable position, which suggests that it might be a physically acceptable position - a consequence that Wilson will likely not accept. But then, does this mean that epiphenomenalism is a logically incoherent position? Wilson doesn't go as far as saying that epiphenomenalism is incoherent. Instead, she seems to find it simply implausible (pp. 98-9, p. 101, p. 141, and pp. 240-1).

I think it needs to be said that we need to make at least a logical room for epiphenomenalism in the relevant debates. The view that some special-science properties are epiphenomenal is a view that several contributors to the debate have taken seriously, and the fact that the problem of higher-level causation is often invoked to corner emergentism into epiphenomenalism goes to show the dialectical relevance of the view. But more importantly, given Wilson's endorsement of metaphysical neutrality regarding powers, causation, properties, and laws, we should be open to the possibility that some accounts of these concepts might make

\footnotetext{
${ }^{5}$ See Morris (2016) for an argument that physicalists should find protophenomenal properties objectionable.

${ }^{6}$ See Baysan (2020) for a detailed treatment of several variants of epiphenomenalism.
} 
epiphenomenalism an empirical possibility. Perhaps the correct theory of causation will imply that there are epiphenomenal properties in nature.

One of the things that Wilson says in justifying her dismissal of epiphenomenalism is that epiphenomenalism is "unsystematic, given that no other empirical goings-on are taken to be epiphenomenal” (p. 241). But I don't find this reason very convincing. If epiphenomenalism is true, causally inefficacious properties are a systematic feature of the natural world. This would be particularly true if epiphenomenalism followed from some assumptions about causation, like that of Non-Overdetermination. If epiphenomenalism about (some) mental properties were true, it would be reasonable to expect to find epiphenomenal properties in various special sciences.

This is not a fatal problem for Wilson's project. Instead, it is something that I would like to hear more about. Similar to what I said in $\$ 3$ in relation to Russellian monism, examining epiphenomenalism with the theoretical tools that Wilson offers in Metaphysical Emergence can only be fruitful in our understanding of emergence.

\section{Concluding Remarks}

Let me conclude by urging that Metaphysical Emergence is a very important contribution to the contemporary literature on the metaphysics of emergence. It is systematic; in fact, it is the most systematic treatment of metaphysics of emergence that I know of. It is very thorough: Wilson complements many of her proposals by discussing and responding to various objections. It is ambitious: while Chapter 7 develops a novel approach to the relationship between conceivability and possibility to tackle epistemic and modal arguments against physicalism, Chapter 8 makes a case for libertarianism about free will. Overall, this is a work of very high quality that we will talk about for many years to come. ${ }^{7}$

\section{REFERENCES}

Baysan, U. 2018. Epiphenomenal properties. Australasian Journal of Philosophy, 96:3, 419431.

Baysan, U. 2020. Rejecting epiphobia. Synthese, 1-19.

Chalmers, D. 2003. Consciousness and its place in nature. In S. P. Stich \& T. A. Warfield (eds.), Blackwell Guide to the Philosophy of Mind (Blackwell).

Chalmers, D. 2006. Strong and weak emergence. In P. Clayton and P. Davies (eds.), The reemergence of emergence (Oxford: Oxford University Press, pp. 244-55).

Chalmers, D. 2015. Panpsychism and Panprotopsychism. In T. Alter \& Y. Nagasawa (eds.), Consciousness in the Physical World: Perspectives on Russellian Monism (Oxford: Oxford University Press).

Christensen, J. \& Baysan, U. 2018. Why incompatibilism about mental causation is incompatible with non-reductive physicalism. Inquiry, 1-23.

\footnotetext{
${ }^{7}$ I am grateful to Luca Barlassina and Neil McDonnell for comments on a previous version of this article, and to Alex Moran and Jessica Wilson for discussion of related content.
} 
List, C. \& Menzies, P. 2009. Nonreductive physicalism and the limits of the exclusion principle. Journal of Philosophy 106, 475-502.

Kim, J. 1998. Mind in a Physical World. Cambridge, MA: MIT Press.

Kim, J. 2003. Blocking causal drainage and other maintenance chores with mental causation. Philosophy and Phenomenological Research, 67: 151-176.

Melnyk, A. 2006. Realization-based formulations of physicalism. Philosophical Studies, 131: 127-55.

Morris, K. 2016. Russellian physicalism, bare structure, and swapped inscrutables. Journal of Consciousness Studies, 23:180-198.

Noordhof, P. 2003. Not old ... but not that new either: Explicability, emergence and the characterisation of materialism. In S. Walter \& H. Heckman (eds.) Physicalism and mental causation (Exeter, Imprint Academic, pp. 85-108).

Wilson, J, 2011. Non-reductive realization and the powers-based subset strategy. The Monist, 94: 121-154.

Wilson, J. 2015. Metaphysical emergence: weak and strong. In T. Bigaj \& C. Wuthrich (eds.), Metaphysics in Contemporary Physics: Poznan Studies in the Philosophy of the Sciences and the Humanities, 251-306.

Wilson, J. 2021. Metaphysical Emergence. Oxford: Oxford University Press. 